\title{
Die Macht von Social Media - Einfluss auf die Reputation von Ärzten und Kliniken
}

\author{
In der Internet-Suche werden Kommentare und Beurteilungen zu Dienstleistern im \\ Gesundheitswesen aus diversen Social-Media-Diensten oft prominenter angezeigt \\ als die offizielle Website. Diese digitale Identität und die Online-Reputation beein- \\ flussen den realen Erfolg.
}

Daniela A. Caviglia

Medienfachfrau und Kommunikatorin
Korrespondenz: Daniela A. Caviglia Präsenz \& Effizienz Steinhusen 5 CH-6114 Steinhuserberg

caviglia[at]praesenz-effizienz.ch
Wie eine Klinik oder Arztpraxis bei Suchmaschinen platziert ist und welchen Eindruck diese Präsenzen vermitteln, entscheidet oft mit über den wirtschaftlichen Erfolg von Kliniken und Praxen. Und diese Tendenz nimmt zu. Smartphone-Apps und die Internet-Suche auf mobilen Endgeräten zeigen in den seltensten Fällen offizielle Websites an, denn Bewertungs- und Suchportale geben eigene Apps heraus und umgehen so die Informationen, die Spitäler und Ärzte von sich aus mit dem weltweiten Netz teilen. Diesen Trend kann man - so praktisch es auch wäre nicht ignorieren.

\section{Die Social-Media-Kette beginnt in der realen Welt}

Alles, was online stattfindet, hat seinen Ursprung in der Offline-Welt, im realen Hier und Jetzt. Die digitale Identität wird vom Arzt, der Ärztin selbst initiiert, auch wenn er oder sie absolut nicht internetaffin ist. Die fachliche Kompetenz, das menschliche

\section{Abbildung 1}

Faktoren, welche die Online-Reputation von Spitälern und Ärzten beeinflussen. Der Grundstein dazu wird in der realen Welt gelegt.

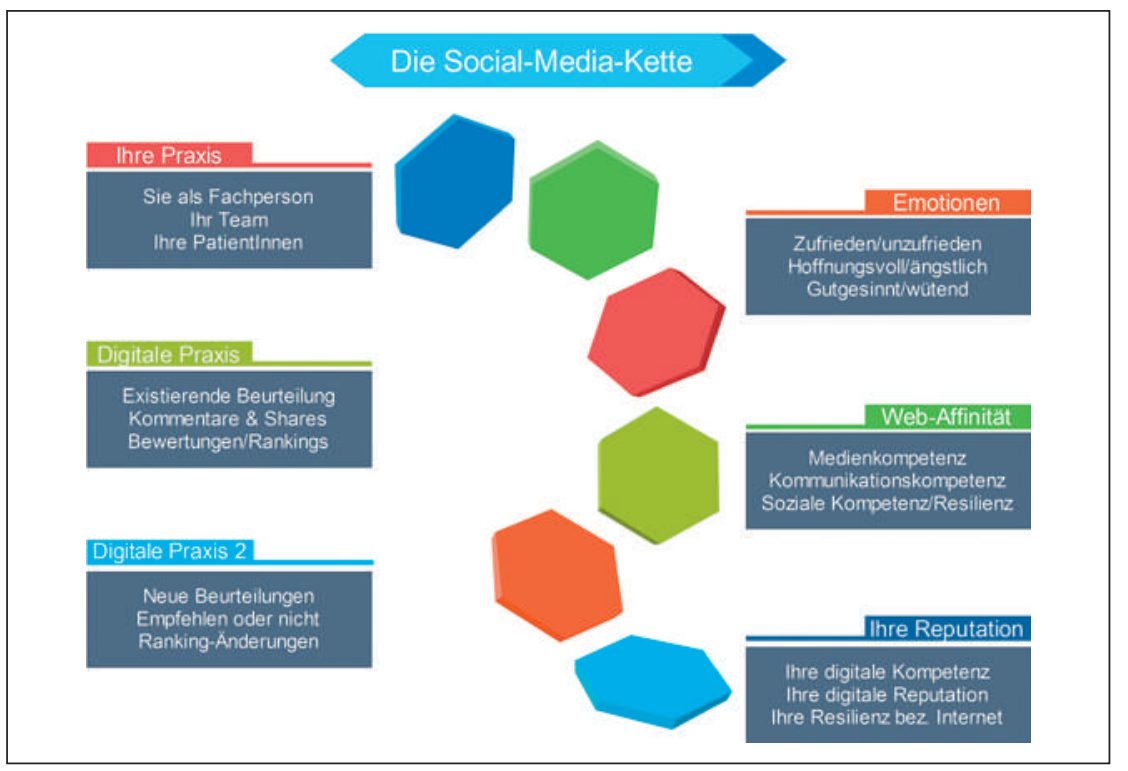

\section{Le pouvoir des médias sociaux -} influence sur la réputation des \section{médecins et des cliniques}

Compte tenu de l'intérêt élevé de la population pour Internet et de l'essor de l'Internet mobile, les médias sociaux revêtent toujours plus d'importance dans des domaines qui avaient jusqu'ici la possibilité de rester en retrait, comme les cabinets médicaux et les hôpitaux.

Sur Google, les commentaires et les évaluations publiés sur les médias sociaux sont souvent mieux placés que les sites officiels des prestataires de santé. Cette identité numérique influence aussi bien le nombre de nouveaux patients que leur attitude au premier contact. Ce qui peut avoir des répercussions positives mais aussi négatives.

Verhalten, allein schon die Entscheidung, als Arzt oder Ärztin tätig zu werden, ist der Keim zur OnlineIdentität. Das allernächste Umfeld - Praxisteam, Klinikumgebung und Patient(inn)en - sind ebenfalls zur «Keimzelle» oder dem ersten Kettenglied zuzurechnen. Das zweite Glied sind die Emotionen. Sie bestimmen die Richtung, welche die Online-Aktivität der Betroffenen einschlägt. Beeinflusst wird diese Richtung ein wenig von bereits bestehenden digitalen Identitäten. Sind vorwiegend positive Bewertungen zu einem Arzt oder einem Spital vorhanden, ist die Hemmschwelle, einen durchwegs negativen Kommentar zu hinterlassen, höher. Das gilt leider auch im umgekehrten Fall.

\section{Reputation, Kompetenz und Resilienz}

Auf digitaler Ebene umfasst das vierte Glied die Kompetenzen der Praxisbetreiber respektive der Klinikleitung. Verfügen sie über Medienkompetenz, um Be- 
wertungen schnell und richtig kommentieren oder verdanken zu können, weisen sie genug Kommunikations- und soziale Kompetenz auf, um auch in heiklen Situationen richtig zu reagieren? Natürlich sind die zwei letzten Kompetenzen bereits in der Offline-Welt also dem ersten Kettenglied - äusserst wirksame Beeinflusser der Social-Media-Kette. Jede neue Beurteilung, verbesserte oder verschlechterte Bewertungen, beeinflussen wiederum die Wachstumsrichtung der digitalen Identität. Und bilden letztlich ein OnlineImage, das sich aus Reputation, Kompetenz und Resilienz zusammensetzt. Ist das Image positiv, wirkt es sich ebenso auf die Realität aus. Nicht nur bezüglich Zahl der Neuzugänge bei Patienten und Stellenbewerbern, sondern auch mit einer verbesserten Grundstimmung bei Erstkontakten, einem emotionalen Bonus des Gegenübers.

\section{Schutz von Patienten -}

\section{vor sich selbst und anderen}

Ein zweites Problem von Social Media betrifft Patientinnen und Patienten. Eine kurze Suche auf Facebook (man muss wirklich nicht lange suchen!) fördert unglaublich viele Veröffentlichungen zutage, bei denen sich einem die Haare sträuben. Säuglinge werden am Tag ihrer Geburt inklusive Namensschild in der Krankenhauswiege fotografiert und öffentlich online gestellt. Tödliche Krankheiten werden mit Details zu

\section{Abbildung 2}

Die Kernpunkte der Team- und Patienten-Aufklärung. Die Rechte und die Privatsphäre Dritter sollten möglichst gut geschützt werden.

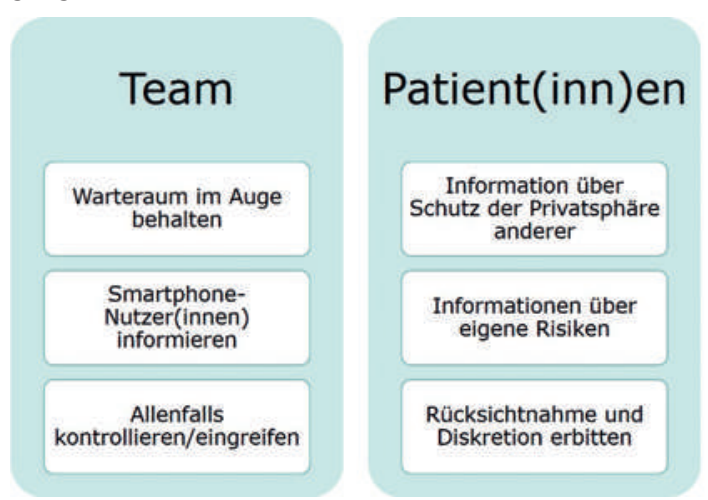

den Betroffenen oder von den Betroffenen selbst verbloggt, Hintergrundinformationen zu Ärzten, Mitpatienten, Pflegepersonal und Details aus den Krankenakten inklusive. «Psychisch krank ist keine Schande» formiert sich als öffentliche Facebookgruppe statt einer diskreten Selbsthilfegruppe in der echten Welt, die Seite «Chronisch krank» veröffentlicht private Krankengeschichten zwischen News aus Medizin und Forschung.

\section{Abbildung 3}

Die Online-Reputation beeinflusst sowohl den realen Geschäftserfolg als auch die Grundstimmung von neuen Patienten beim Erstbesuch, wenn sie die Praxis oder Klinik über das Internet gefunden haben.

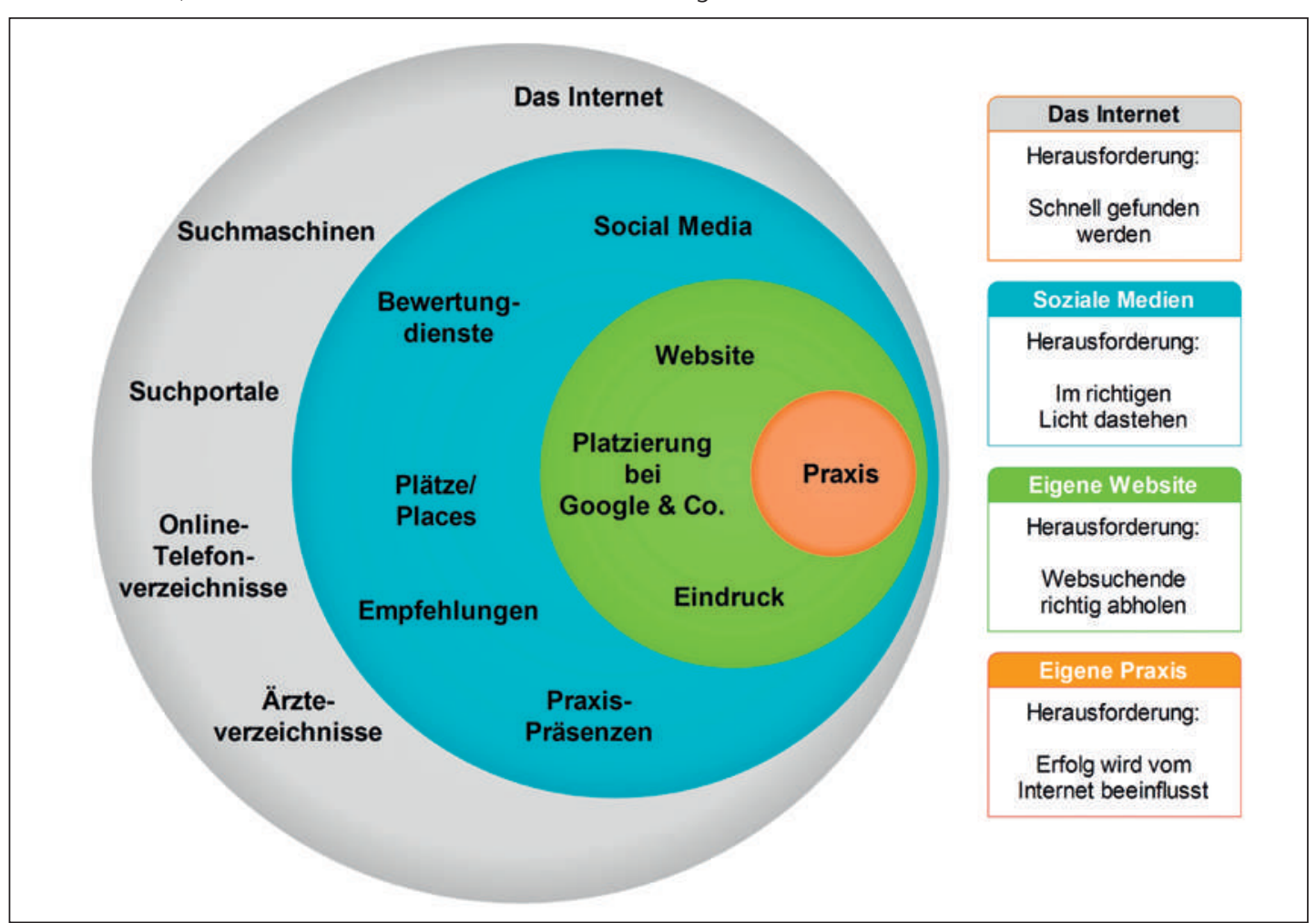




\section{Aufklären, aufklären und nochmals aufklären}

Auch wenn prinzipiell jeder Internetnutzer und jede Internetnutzerin selber entscheiden sollte, wo die Hemmschwelle beim Veröffentlicher privater Details liegt, so liegt eine gewisse Aufklärungspflicht auch beim jeweiligen Umfeld. Beispielsweise zum Schutz der betroffenen Drittpersonen. Ein Neugeborenes kann nicht entscheiden, ob es im Alter von wenigen Stunden schon auf Facebook präsent sein will. Und auch viele Betroffene der anderen Veröffentlichungen werden ihr Einverständnis kaum gegeben haben. Auf die Privatsphäre dieser Drittpersonen hinzuweisen, ist nicht mehr als sinnvoll. flusst werden. Die Optimierung beginnt mit Recherche. Wo überall ist die Klinik oder Praxis präsent? Welche Kommentare haben Patientinnen und Patienten hinterlassen? Die meisten Online-Präsenzen, die durch solche Patienten-Einträge entstehen, wie sie bei kununu.com oder facebook.com sowie durch Betreiber von Suchportalen wie comparis.ch, plus. google.com oder doktor.ch erstellt werden, können beantragt und anschliessend bearbeitet werden. Der Vorteil: Zukünftig erhält man eine Benachrichtigung, wenn neue Beurteilungen und Kommentare von Patienten veröffentlicht werden. Und man kann zufriedene Patientinnen und Begleitpersonen aktiv um Bewertungen bitten. Erfahrungsgemäss wirken aktiv

\section{«Die Online-Reputation beeinflusst sowohl den realen Geschäftserfolg als auch die Grundstimmung von neuen Patienten.»}

Im Wartezimmer einer Arztpraxis vertreibt man sich die Zeit. Und heutzutage in vielen Fällen auf Facebook und Co. Verbote nützen angesichts neuer Produkte wie Smartwatches oder Google Glass, also Uhren und Brillen mit Smartphone-Funktionen, nicht mehr viel. Aufklärung dafür umso mehr. Das Praxis- oder Klinik-Team sollte entsprechend geschult, für Patienten allenfalls Merkblätter aufgelegt oder gut sichtbar angebracht werden.

\section{Wie die Online-Reputation beeinflusst werden kann}

Für Praxenbetreiber, Fachärzte und Spitäler gilt gleichermassen: Die Online-Reputation beeinflusst den realen Erfolg. Sie kann aber auch ihrerseits beein- betreute und ansprechend gestaltete Internetpräsenzen um einiges positiver auf Webnutzer und mögen auch zu einem gewissen Teil emotionale Wogen zu glätten. Die Basis für eine verbesserte Online-Reputation. Und bessere Erfolgschancen in der realen Welt.

\section{Interaktiver Artikel}

Wollen Sie diesen Artikel kommentieren? Nutzen Sie dafür die Kommentarfunktion in der OnlineVersion oder sehen Sie nach, was Ihre Kolleginnen und Kollegen bereits geschrieben haben: www.saez.ch/aktuelle-ausgabe/interaktive-beitraege/ 